\title{
Optimized Lane Assignment Using Inter-Vehicle Communication
}

\author{
Thanh-Son Dao, Christopher Michael Clark and Jan Paul Huissoon
}

\begin{abstract}
This paper presents an approach to lane assignment for highway vehicles that increases traffic throughput while ensuring they exit successfully at their destinations. Most of current traffic management systems do not consider lane organization of vehicles and only regulate traffic flows by controlling traffic signals or ramp meters. However, traffic throughput and efficient use of highways can be increased by coordinating driver behaviors intelligently. The goal of this research is to form a distributed control strategy for cars themselves to select lanes using inter-vehicle communication. Initial results are promising and demonstrate that intelligent lane selection can decrease vehicle traffic time.
\end{abstract}

\section{INTRODUCTION}

To define the next-generation of land transportation systems, several transportation projects have been initiated. Examples include the California Partners for Advanced Transit and Highway (PATH) program, the AUTO21 project and other Intelligent Transportation System (ITS) programs. The goals of these programs are quite broad and include increased traffic throughput, less accidents, reduced fuel consumption and better driving experience. However, very little research has been done on developing appropriate algorithms that allow cars to sense and intelligently affect the traffic flows that could result in more efficient use of highways.

The work in this paper targets the problem of traffic management with the effort to develop scheduling and control techniques to support (semi)-autonomous driving on urban multi-lane highways with multiple entry (on-ramp) and exit (off-ramp) points. Advanced traffic management systems are designed to reduce congestion and increase overall traffic capacity. Currently, almost all such systems regulate traffic flow by controlling traffic signals or highway ramp meters. In these systems, the traffic is treated as a single mass and the behaviors of individual cars are normally ignored [1][2][3]. Most recently, Goolsby et al. [4] used changeable lane assignment signs at frontage road intersections to adapt to changing traffic conditions at different times of day. When these interchanges experience high turning movement demands, permitted double turns are often used to increase traffic throughput. This approach misses an important component of traffic management: coordination of cars themselves.

This work is funded by AUTO21 Canada.

T. S. Dao is a PhD candidate at the Lab for Autonomous and Intelligent Robotics, Dept. of Mechanical Engineering, University of Waterloo, ON, Canada N2L 3G1 tsdaodengmail. uwaterloo. ca

C. M. Clark is an Assistant Professor at the Computer Science Department, California Polytechnic State University, San Luis Obispo, CA, USA 93407 cmclarkecalpoly.edu

J. P. Huissoon is a Professor at the Dept. of Mechanical Engineering, University of Waterloo, ON, Canada N2L 3G1 jph@mecheng1. uwaterloo.ca
One of the first works on collaborative lane assignment was conducted by Hall [5] who considered the lane-change maneuvers in deriving a min-max optimization problem. The objective was to equalize the workload and congestion across lanes. However, this work assumed that the vehicles flow into or out of highway at any point as in fluid flow (flux) systems and exact entrance and exit locations were not modeled. This work also does not deal with a distributed control strategy for individual vehicles.

Medanic et al. [6] defined a lane assignment problem using the origin-destination matrix, with the objective of reducing the unnecessary crossings on the highway. Their lane assignment strategy assigned a lane to each vehicle based on its origin and destination. The simulation results showed the effects of maneuvers on costs, but a distributed control strategy for individual car was not presented.

Hall et al. [7] presented another approach to the lane assignment problem. They used tools from network theory to represent a highway with arcs and nodes. The network representation of a highway in their work has potential in modeling traffic in highway systems. However, their paper does not deal explicitly with the lane change effect on the highway capacity.

Kim et al. [8] used the same approach by partitioning the highway systems. In their work, the lane assignment problem was formulated as an optimization problem to find proper positions of partitions on an itinerary matrix. Then the optimization problem was solved using genetic algorithms. The validation of the algorithm was conducted by comparing it with a random strategy in a realistic situation.

Lane selection behaviors among cars could be coordinated to achieve greater traffic throughput. A challenge to this problem lies in the development of a cooperative driving strategy for traffic management. This research explores the problem of optimization of lane selection. Our fundamental assumption is that the cars are equipped with microprocessors, GPS receivers, and wireless communication devices. A following assumption is that any two vehicles within a certain radius of each other can communicate. A car should select lanes not only to improve its own travel time, but the travel time of other cars and the overall traffic flow of the highway system.

Several topics in regard to inter-vehicle communication, lane finding and lane assignment are explored in this paper: First, a brief introduction about our communication method is given in Section II. The system architecture and the proposed steps to the problem are discussed in Section III. Section IV deals with flow rates estimation. Section V discusses a distributed control strategy for routing cars to 
appropriate lanes while satisfying some constraint conditions. A proposed cost function to optimize is also discussed. To accurately model traffic flow, a car-following model is proposed in Section VI. Currently, by solving a minimization problem the algorithm is able to send cars to appropriate lanes with an effort to balance lane traffic flows and decrease the vehicles' travel time. Simulations to evaluate the algorithm are provided in Section VII, followed by some concluding remarks in Section VIII.

\section{COMMUNICATION}

In our system, it is assumed that the cars can perceive certain states of surrounding cars, i.e., their positions, destinations and speeds. The system could receive this directly from other cars via radio waves or an ad hoc network.

Fig. 1 illustrates the communication of cars in a specific situation. Cars 2, 3 and 4 are within communication range and they can inter-change information with one another while car 1 can only communicate with car 2 . This means that the cars can only have a partial view of the overall traffic condition. Another option is to give all of the cars complete information regarding all other cars by letting them pass back their own information and the information of other cars. For example, by passing back car 1's information to car 3 and 4 , the last two cars will have a clearer picture of the traffic situation. In this way, the last car is the one that has the most information of the system.

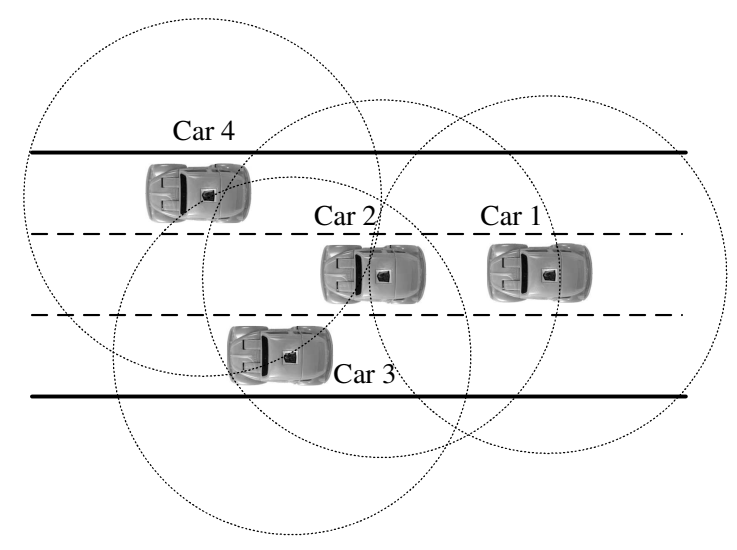

Fig. 1. Inter-vehicle communication.

In our work, we use low-cost sensors and propose a popular communication method: GPS and wireless ad-hoc networks. The GPS is the most convenient method for determining car position in a global coordinate system. A detailed description of GPS is provided by Farrel and Barth [9]. For standard GPS with large measurement noise, a filter can be used to get more accurate vehicle positions [10]. The architecture for the system is shown in Fig. 2. Each car is equipped with a GPS receiver, a processor to implement lane routing algorithm and and a device to communicate with other cars.

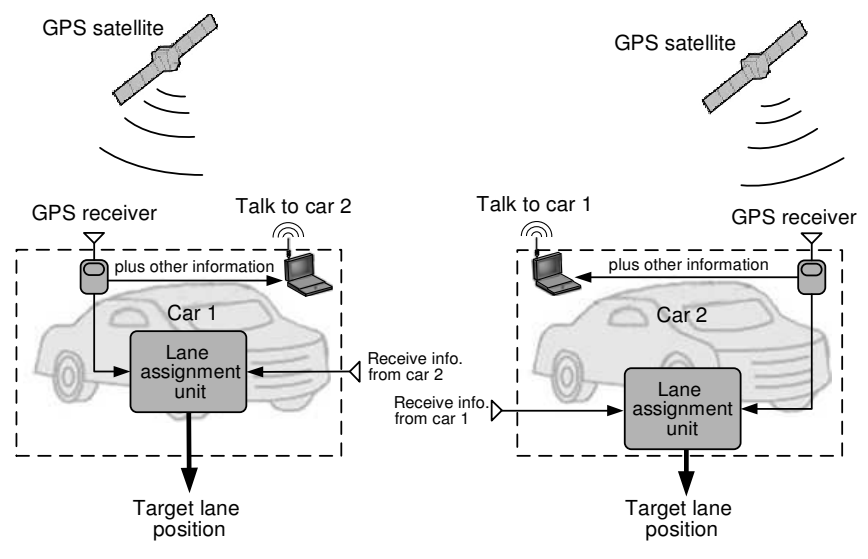

Fig. 2. Architecture for inter-vehicle communication.

\section{SYSTEM ARCHITECTURE}

There are two architectures for implementing lane selecting schemes: centralized and decentralized. The key advantage of decentralized over centralized approach is scalability. It is easier to grow a decentralized system and to add new elements to it.

In this paper, the lane assignment problem for a highway with $n_{e}$ entry (on-ramp) and $n_{d}$ exit (off-ramp) points intended for (semi)-autonomous driving is considered, each lane is characterized by a different nominal driving speed. The highway system used in this work is discretized into segments. For every on-ramp, a new segment is created which contains one or more lanes. The number of lanes can vary from segment to segment but must be a constant along each segment. Lanes are numbered from right to left, with the right-most lane numbered 1. On-ramps and off-ramps are designated as lane 0 . Lane exits and lane entrances are assumed to occur on the right side of the highway. It is assumed that a typical vehicle would enter the lanes and proceed gradually to the lane assigned to it.

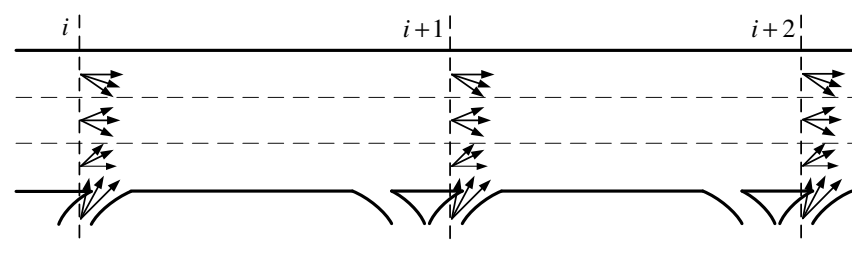

Fig. 3. Highway system.

As illustrated in Fig. 3, the highway is represented by a network. Nodes are assigned to the start of each entry point. The objective is to maximize the flow across the highway. Mathematically speaking, the constraints of the problem are:

1) Non-negativity: the number of cars can not be negative,

2) Lane capacity: capacity that determines the maximum rate at which vehicles can enter a lane can not be exceeded.

The proposed implementation of the overall problem can be presented by a closed-loop system and will be broken 


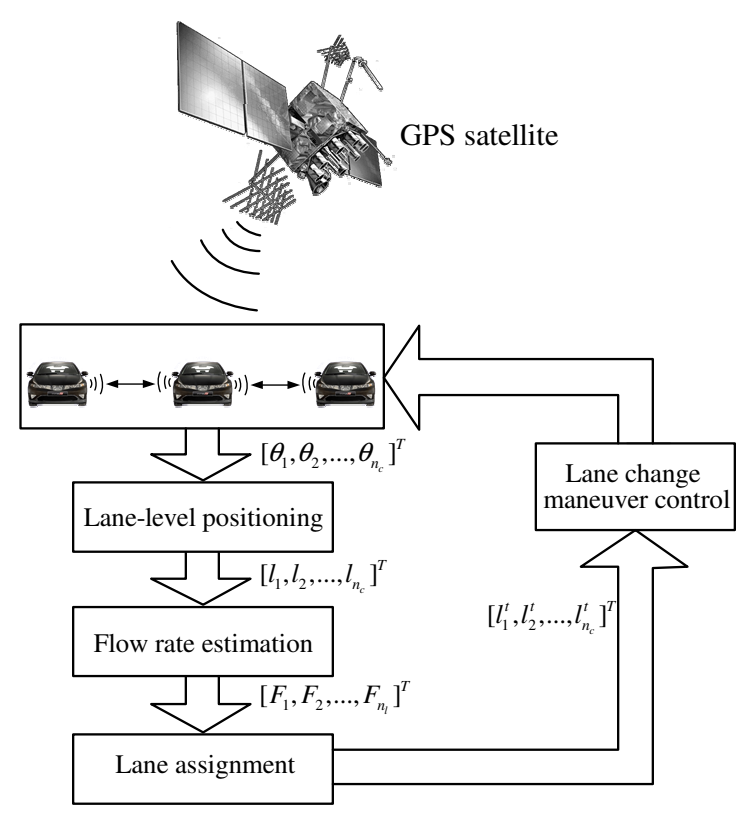

Fig. 4. Control diagram: $\left[\theta_{1}, \theta_{2}, \ldots, \theta_{n_{c}}\right]^{T}$ are the GPS measurements for car 1 through car $n_{c},\left[l_{1}, l_{2}, \ldots, l_{n_{c}}\right]^{T}$ are their lane positions, $\left[F_{1}, F_{2}, \ldots, F_{n_{l}}\right]^{T}$ are the estimated flows in lane 1 through lane $n_{l}$, and $\left[l_{1}^{t}, l_{2}^{t}, \ldots, l_{n_{c}}^{t}\right]^{T}$ are target lanes for these cars.

into three main parts (see Fig. 4): (1) lane positioning (lane occupancy estimation), (2) lane flow estimation, and (3) lane assignment. Note that each of these three individual steps is accomplished through the collaboration of multiple vehicles communicating within ad hoc networks.

Lane positioning is necessary for cars to know where they are on the highway. In an automated highway system, cars are expected to know the lanes they occupy. This serves as a basis for the rest of lane assignment algorithm. A lane positioning algorithm was discussed in detail in previous work [10]. The lane assignment strategy must also satisfy a number of constraints, including lane capacity. The next section will show how lane flow estimation can be implemented.

\section{FLOW RATE ESTIMATION}

In order to know if a lane capacity has been exceeded, the traffic flow rate in each lane must be estimated. This can be accomplished by counting the number of cars passing through a certain point. Our approach is to use inter-vehicle communication. The strategy for flow rate estimation is illustrated in Fig. 5. The time instances $t_{1}$ and $t_{2}$ are the time that car 1 and car 2 hit point $P$, respectively. The instantaneous flow rate as car 2 passes point $P$ is: $f_{P, n}=\frac{1}{t_{2}-t_{1}}$. The denominator $t_{2}-t_{1}$ is called the time headway for car 2 . The current flow can be calculated recursively by fusing $f_{P, n}$ with the flow in the last time step

$$
F_{P, n}=\frac{f_{P, n}+(n-1) F_{P, n-1}}{n},
$$

where $n$ is the number of cars passing through point $P$; the factors $\lambda=\frac{1}{n}$ and $\mu=\frac{n-1}{n}$ are considered as weight factors.
As the number of vehicles grows large, i.e., $n \rightarrow \infty$, Eq. (1) will be more likely to convert to the cumulative flow in previous time steps. To avoid this, a sliding window that defines an amount of cars stored in the counting buffer can be used. As the cars are being counted, buffer space is freed up to accept more inputs.

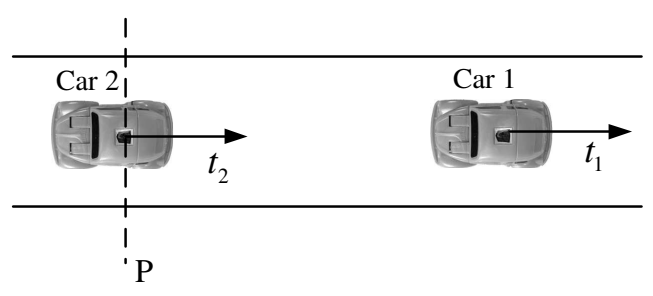

Fig. 5. Flow rate estimation.

\section{LANE ASSIGNMENT OPTIMIZATION}

In this Section, a direction for lane assignment is proposed with an aim to enhance traffic system operation, enhance safety, reduce travel time and improve traffic quality. The lane optimization problem is formulated here as a linear programming problem [11].

Since it is not possible for cars to communicate with all other cars on the highway via ad hoc wireless network, it is necessary to pick out a subset of cars that are able to talk to one another for cooperative planning. The current approach is to define local clusters of vehicles near the vicinity of road segments. Given a group of cars within the vicinity (defined by $\Delta$ as in Fig. 6) of a road segment, they communicate with each other to create a plan for optimizing traffic throughput in the upcoming road segment (segment $i$ ). For illustration, call the group of cars within $\Delta$ group $A$ (shown in a bright color) and the flowing cars that will be within $\Delta$ in the next lane assignment group $B$ (shown in a dark color). As the first car in group A hits the start of segment $i$, they talk to each other to determine which cars are within $\Delta$. The lane assignment algorithm is then implemented by individual cars in group A. Once cars in a group have been assigned lanes, the cars will not be reassigned until they form a new group at the vicinity of the next segment. Fig. 6 (bottom) illustrates the lane assignment for cars in group B when the first car in this group hits the start of segment $i$.

The lane optimization problem in our work is formulated as follows: Let us consider an $n_{l}$-lane highway system as shown in Fig. 3 where $i, i+1, i+2, \ldots$, indicate the road segment numbers starting at the entry point positions. Let the distance between entry $i$ and exit $j$ be $d_{i, j}$ and the nominal velocity on lane $l$ be $v^{l}$. The estimated time that takes one car currently in lane $l_{c}$ to switch to lane $l$, then travel from $i$ to exit $j$ is $\frac{d_{i, j}}{v^{l}}+T_{l_{c}, l}$ where $T_{l_{c}, l}=\tau\left|l-l_{c}\right|+\tau l$ is the time delay caused by lane change maneuvers considering that the maneuver cost (time penalty) for a car to move from lane $l_{c}$ to lane $l$ is $\tau\left|l-l_{c}\right|$ and from lane $l$ to an off-ramp (exiting) is $\tau l$, where $\tau$ is the predefined time constant (offramps are considered as lane 0 ). The total time of all cars 


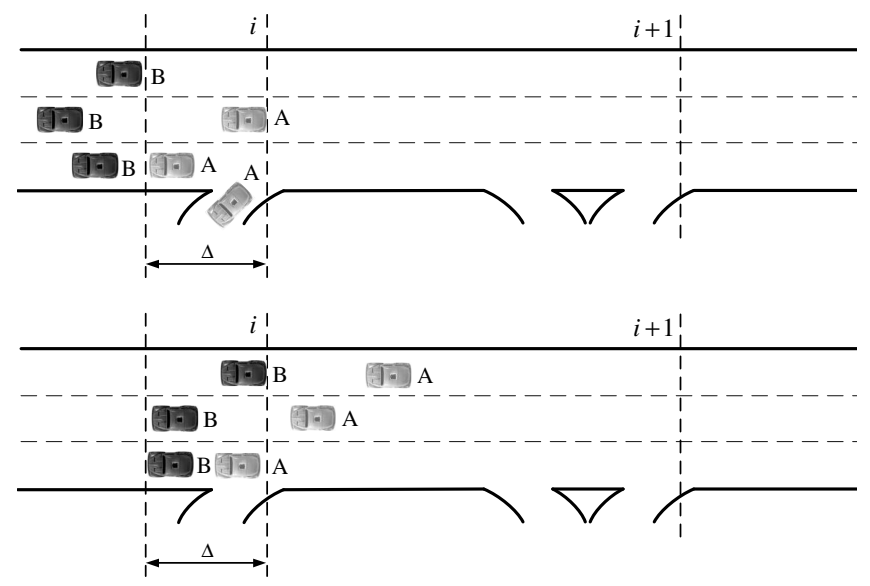

Fig. 6. Lane assignment strategy: lane assignment for group A (top) and group B (bottom).

in lane $l_{c}$ starting at segment $i$ is $\sum_{j=i+1}^{n_{d}} N_{i, j}^{l_{c}, l}\left(\frac{d_{i, j}}{v^{l}}+T_{l_{c}, l}\right)$, where $N_{i, j}^{l_{c}, l}$ is the number of cars currently in lane $l_{c}$ that will be traveling from the start of segment $i$ to exit $j$ in lane $l$, and $n_{d}$ is the number of exit points. Thus a candidate for the cost function to be minimized is the total travel time, $\sum_{l_{c}=0}^{n_{l}} \sum_{l=1}^{n_{l}} \sum_{j=i+1}^{n_{d}} N_{i, j}^{l_{c}, l}\left(\frac{d_{i, j}}{v^{l}}+T_{l_{c}, l}\right)$.

The problem is to assign lanes to all cars within $\Delta$ at the start of each segment. Let $x_{i, j}^{l_{c}, l}$ be the percentage of cars (within $\Delta$ ) traveling from $i$ to $j$ that will be sent from lane $l_{c}$ to lane $l$. The factor $x_{i, j}^{l_{c}, l}$ relates the number of cars $N_{i, j}^{l_{c}, l}$ by $N_{i, j}^{l_{c}, l}=N_{i, j}^{l_{c}} x_{i, j}^{l_{c}, l}$. The cost function for segment $i$ becomes

$$
\Phi_{i}=\sum_{l_{c}=0}^{n_{l}} \sum_{l=1}^{n_{l}} \sum_{j=i+1}^{n_{d}} N_{i, j}^{l_{c}}\left(\frac{d_{i, j}}{v^{l}}+T_{l_{c}, l}\right) x_{i, j}^{l_{c}, l}
$$

The minimization problem can be cast as a linear programming problem to solve for $x_{i, j}^{l_{c}, l}$, s with the cost function in Eq. (2) subject to the following constraints:

1) Non-negativity: $x_{i, j}^{l_{c}, l} \geq 0$,

2) Lane capacities: $\lambda_{i, j}^{l_{c}, l} f\left(x_{i, j}^{l_{c}, l}\right)+\mu_{i}^{l} \hat{F}_{i}^{l} \leq C_{\max }^{l}$, where $f\left(x_{i, j}^{l_{c}, l}\right)=\frac{N_{i, j} x_{i, j}^{l_{c}, l}}{\delta_{i, j}^{l, l}}$ is the instantaneous estimated flow which would be caused by the $N_{i, j} x_{i, j}^{l_{c}, l}$ cars, $\delta_{i, j}^{l_{c}, l}$ is the total time headway for the $N_{i, j} x_{i, j}^{l_{c}, l}$ cars, $\hat{F}_{i}^{l}$ is the estimated flow in lane $l, \lambda_{i, j}^{l_{c}, l}$ and $\mu_{i}^{l}$ are the weight factors (see Section IV for weight factors and flow estimation), and $C_{\max }^{l}$ is the capacity of lane $l$,

3) Percentages sum to $1: \sum_{l=1}^{n_{l}} x_{i, j}^{l_{c}, l}=1$.

One should keep in mind that in all constraint conditions, the superscript $l_{c}$ takes on values from 0 to $n_{l}$, i.e., $0 \leq$ $l_{c} \leq n_{l} ; 1 \leq l \leq n_{l}$; and $i+1 \leq j \leq n_{d}$. Therefore, there exists $n_{l}\left(n_{l}+1\right)\left(n_{d}-i\right)$ equations for constraints 1 and 2 , and $\left(n_{l}+\right.$ 1) $\left(n_{d}-i\right)$ equations for constraint 3 .

To summarize, the constrained minimization problem is of the form

$$
\min \mathbf{c}^{T} \mathbf{x}
$$

subject to

$$
\mathbf{A x} \leq \mathbf{b}, \mathbf{A}_{e q} \mathbf{x}=\mathbf{b}_{e q}, \text { and } \mathbf{x} \geq \mathbf{0} .
$$

The $n_{l}\left(n_{l}+1\right)\left(n_{d}-i\right) \times 1$ vector $\mathbf{c}$ has the form

$$
\begin{aligned}
& \mathbf{c}=\underbrace{\left[N_{i, i+1}^{0}\left(\frac{d_{i, i+1}}{v^{1}}+T_{0,1}\right) \ldots N_{i, i+1}^{0}\left(\frac{d_{i, i+1}}{v^{n_{l}}}+T_{0, n_{l}}\right)\right.}_{n_{l} \text { components }} \ldots \\
& \ldots \underbrace{N_{i, n_{d}}^{n_{l}}\left(\frac{d_{i, n_{d}}}{v^{1}}+T_{n_{l}, 1}\right) \ldots N_{i, n_{d}}^{n_{l}}\left(\frac{d_{i, n_{d}}}{v^{n_{l}}}+T_{n_{l}, n_{l}}\right)}_{n_{l} \text { components }}]^{T},
\end{aligned}
$$

vector $\mathbf{x}$ is

$$
\mathbf{x}=[\underbrace{x_{i, i+1}^{0,1} \ldots x_{i, i+1}^{0, n_{l}}}_{n_{l}} \cdots \underbrace{x_{i, n_{d}}^{n_{l}, 1} \ldots x_{i, n_{d}}^{n_{l}, n_{l}}}_{n_{l}}]_{n_{l}\left(n_{l}+1\right)\left(n_{d}-i\right)}^{T},
$$

matrix $\mathbf{A}$ has the form

$$
\mathbf{A}=\left[\begin{array}{ccc}
\frac{\lambda_{i, i+1}^{0,1} N_{i, i+1}^{0}}{\delta_{i, i+1}^{0,1}} & & \\
& \ddots & \\
& & \frac{\lambda_{i, n_{d}}^{n_{l}, n_{l}} N_{i, n_{d}}}{\delta_{i, n_{d}}^{n_{l}, n_{l}}}
\end{array}\right],
$$

and $\mathbf{A}_{e q}$ is a band matrix

$$
\mathbf{A}_{e q}=\left[\begin{array}{ccc}
\overbrace{1 \cdots 1}^{n_{l}} & & \\
& \ddots & \\
& & \overbrace{1 \cdots 1}^{n_{l}}
\end{array}\right] .
$$

Matrices $\mathbf{A}$ and $\mathbf{A}_{e q}$, which are corresponding to the constraints 2 and 3 , are $\left[n_{l}\left(n_{l}+1\right)\left(n_{d}-i\right)\right] \times\left[n_{l}\left(n_{l}+1\right)\left(n_{d}-i\right)\right]$ and $\left[\left(n_{l}+1\right)\left(n_{d}-i\right)\right] \times\left[n_{l}\left(n_{l}+1\right)\left(n_{d}-i\right)\right]$ matrices respectively.

Vectors $\mathbf{b}$ and $\mathbf{b}_{e q}$ are

$$
\mathbf{b}=\left[\begin{array}{c}
C_{\max }^{1}-\mu_{i}^{1} \hat{F}_{i}^{1} \\
\vdots \\
C_{\max }^{n_{l}}-\mu_{i}^{n_{l}} \hat{F}_{i}^{n_{l}}
\end{array}\right]_{n_{l}\left(n_{l}+1\right)\left(n_{d}-i\right)},
$$

and

$$
\mathbf{b}_{e q}=\left[\begin{array}{c}
1 \\
\vdots \\
1
\end{array}\right]_{\left(n_{l}+1\right)\left(n_{d}-i\right)}
$$

This problem can be solved by the Simplex algorithm with the flow rate for each lane being estimated using the strategy given in the Section IV. The numbers of vehicles, which are integers, are obtained by rounding $N_{i, j} x_{i, j}^{l_{c}, l}$ to the nearest integers after solving the optimization problem. The optimization algorithm tends to assign a faster lane to vehicles which travel longer distance. 


\section{SPEED-FLOW-DENSITY RELATIONSHIP}

The total travel time can be better estimated by taking the speed-flow-density relationship into account. To formulate Eq. (2), the vehicle speeds are assumed to be some nominal lane velocities. However, in an actual highway, the vehicle speed is dependent on the traffic density (and hence the flow rate).

In this work, Van Aerde's model [12] is adopted and implemented in the traffic simulator to model the speedflow-density relationship. This integration model that uses a steady-state car-following model proposed by Van Aerde combines of the Pipes and Greenshields models [13] into a single-regime model. The model, which requires three input parameters, can be calibrated using field loop detector data. The efforts for calibrating Van Aerde's model were described in detail in [12]. The Van Aerde single-regime model overcomes the shortcomings of Greenshields and Pipes models which are often inconsistent with field data from a variety of highways (see [13]).

Van Aerde's model can be described by a series of expressions as follows

$$
\begin{gathered}
\delta=\frac{1}{c_{1}+\frac{c_{2}}{v_{f}-v}+c_{3} v}, \\
m=\frac{2 v_{c}-v_{f}}{\left(v_{f}-v_{c}\right)^{2}}, \\
c_{2}=\frac{1}{\delta_{j}\left(m+\frac{1}{v_{f}}\right)} \\
c_{3}=\frac{-c_{1}+\frac{v_{c}}{C_{\max }}-\frac{c_{2}}{v_{f}-v_{c}}}{v_{c}}
\end{gathered}
$$

where:

$\delta=$ traffic density $(v e h / k m)$ or the inverse of the vehicle headway $(\mathrm{km} / \mathrm{veh})$,

$v=$ vehicle speed $(\mathrm{km} / \mathrm{h})$,

$v_{f}=$ free-speed $(\mathrm{km} / \mathrm{h})$,

$v_{c}=$ speed at capacity $(\mathrm{km} / \mathrm{h})$,

$\delta_{j}=$ jam density $(v e h / k m)$,

$c_{1}=$ fixed distance headway constant $(\mathrm{km})$,

$c_{2}=$ first variable headway constant $\left(\mathrm{km}^{2} / \mathrm{h}\right)$,

$c_{3}=$ second variable headway constant $\left(h^{-1}\right)$.

In practice, the calibration of the car-following model requires the estimation of three parameters: $v_{f}, v_{c}$, and $\delta_{j}$. The vehicle speed can be inferred from traffic density $\delta$ and flow rate $F$ using the fundamental speed-flow-density relationship

$$
v=\frac{F}{\delta} .
$$

From Eq. (3a), the traffic density $\delta$ can be expressed as a function of flow by replacing $v$ with Eq. (4). This gives the quadratic equation

$$
\begin{array}{r}
\delta^{2}\left(c_{1} v_{f}+c_{2}\right)+\delta\left(-c_{1} F+c_{3} F v_{f}-v_{f}\right)+ \\
+\left(F-c_{3} F^{2}\right)=0 .
\end{array}
$$

The estimate vehicle speed $v^{l}$ in Eq. (2) (represented by $v$ in Eqs. (3)-(4)) is a function of flow and can be obtained by solving Eq. (5) and substituting the resulting $\delta$ into Eq. (4). In summary, the procedure is to measure the flow rate $F$ for each lane, then solve for $v$ and use it in the cost function in Eq. (2) instead of the nominal lane velocities.

\section{SIMULATION}

To implement simulations, the VISSIM software package [14] was used. VISSIM is a microscopic, time step and behavior based simulator developed to analyze the full range of functionally classified roadways. It is capable of modeling traffic with various control measures in a $3 D$ environment. VISSIM lets us communicate and control the behaviors of vehicles through a dynamic link library (DLL) file complied from $\mathrm{C} / \mathrm{C}++$ code. Vehicle parameters from the external driver model DLL output function are stored within member variables of a designated vehicle class object. A detailed description about the software architecture used in this research is provided in [15].

Based on the lane flows estimation strategy in Section IV and the minimization of the cost function in Eq. (2), it is possible to assign appropriate lanes to cars. Up to this point, our system assigns lanes to cars once they come within the vicinity of the next road segment. The average travel time of all the cars on the highway is also calculated to evaluate the effectiveness of the algorithm.

The four-lane highway used in the simulation has three entry and three exit points. The capacity for each of the four lanes is $2000 v e h / h$. The highway starts with zero traffic and the vehicles are generated randomly by VISSIM. The speed-flow relationships in all four lanes of the highway are shown in Fig. 7, which are similar to the calibration results from a real freeway described in [12]. The freespeeds for lane 1 through lane 4 are $80 \mathrm{~km} / \mathrm{h}, 90 \mathrm{~km} / \mathrm{h}, 100$ $\mathrm{km} / \mathrm{h}$ and $110 \mathrm{~km} / \mathrm{h}$, respectively. The speeds at capacity $v_{c}$ in the four lanes are $81 \%$ of the free-speeds. To ensure the consistency between simulations with and without lane assignment, the simulation parameters such as speed-flowdensity model (Van Aerde's model), positions of lane change decision, minimum lane acceptance gaps, etc., are the same for all simulations. The only thing making the two strategies different is that vehicles' lane changing behavior is set to "free lane selection" (vehicles can overtake any lanes) mode in simulations without lane assignment.

When a group of cars are within the $\Delta$ of a road segment, they communicate with other cars to plan for optimizing traffic throughput in the current road segment. The strategy given in Section $\mathrm{V}$ appears to be effective in simulations as shown in Fig. 8. These figures show the average travel time for 1000 vehicles on the highway with different total input volumes and the time constant $\tau$ of $10 \mathrm{~s}$. The total volumes 

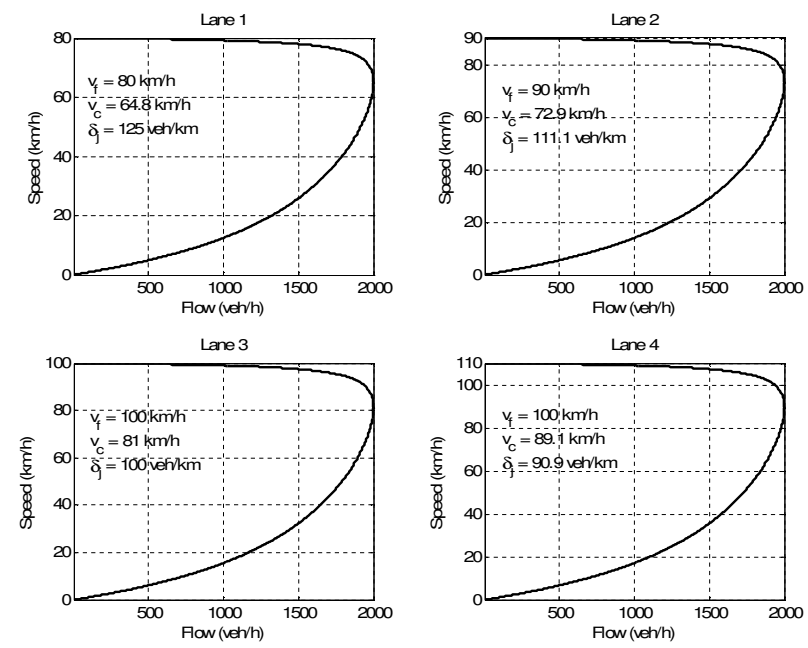

Fig. 7. Speed-flow relationships for simulations.

at the inputs in the three simulations are $2000 \mathrm{veh} / \mathrm{h}, 4000$ $v e h / h$ and $6000 v e h / h$, respectively.

The first simulation (Fig. 8 (top)) shows a large gap (and hence the big improvement) regarding the average traveling times between with and without the implementation of the lane assignment algorithm while the gaps are smaller in the next two simulations (Fig. 8 (middle and bottom)). This can be explained by the fact that when the traffic density gets higher, it is harder to find empty slots in the fast lanes which are filled up very quickly. The initial results show a reduction in the average travel time when the lane assignment algorithm is applied.
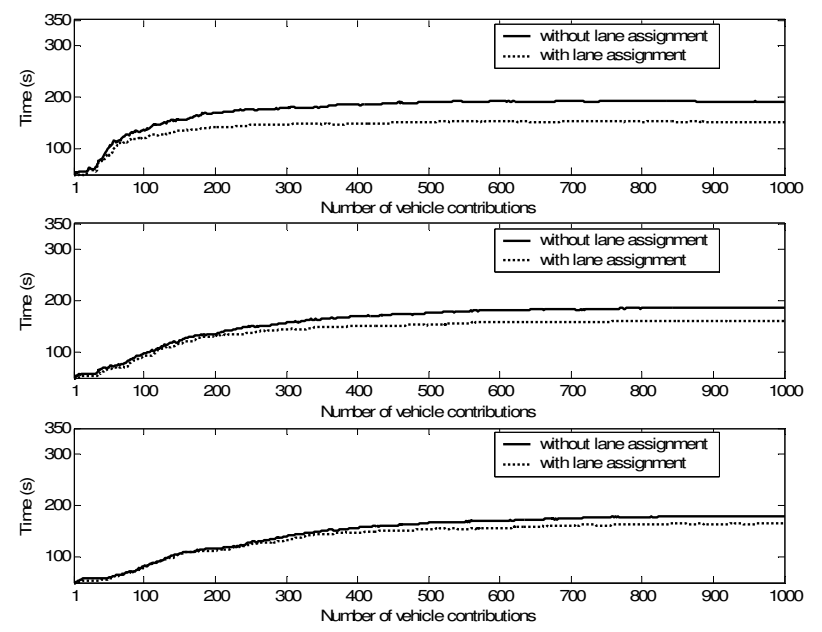

Fig. 8. Improvement over average travel time for 1000 cars with the input volume being $2000 \mathrm{veh} / \mathrm{h}$ (top), $4000 \mathrm{veh} / \mathrm{h}$ (middle), and $6000 \mathrm{veh} / \mathrm{h}$ (bottom).

\section{CONCLUSIONS AND FUTURE WORKS}

An algorithm for optimization of lane assignment was developed with the effort to increase traffic throughput. We have proposed a linear model for lane assignment and presented preliminary results on the use of a linear programming algorithm in the solution of the problem. Initial results are promising and demonstrate that intelligent lane selection can improve highway capacity.

The potential future direction for this work will be on hardware implementation. At the current conditions of intelligent transportation systems, it is challenging to implement the lane assignment algorithm in practice since this method requires a large number of vehicles that are equipped with GPS receivers and communication ability. However, an implementation of the algorithm in a smaller scale can be done by using a number of autonomous mobile robots.

\section{ACKNOWLEDGMENTS}

Financial support for this research has been provided by AUTO21 Canada.

\section{REFERENCES}

[1] J. F. Gilmore, K. J. Elibiary, and H. C. Forbes, "Knowledge-based Advanced Traffic Management System", in Proc. of IVHS America Atlanta, 1994, GA.

[2] K. Kagolanu, R. Fink, H. Smartt, R. Powell, and E. Larson, "An Intelligent Traffic Controller", in Proc. of the Second World Congress on Intelligent Transportation Systems, Japan, 1995, pp. 259-264.

[3] F. .J. Pooran, P. .J. Tarnoff, and R. Kalaputapu, "RT-TRACS: Development of the Real-Time Control Logic", in Proc. of the 1996 Annual Meeting of ITS America, 1996, pp. 422-430.

[4] M. E. Goolsby, D. W. Fenno, and A. P. Voigt, "Project Summary Report 2910-S: Changeable Lane Assignment System (CLAS) on Frontage Roads", Texas Transportation Institute, 2000.

[5] R. W. Hall, "Longitudinal and Lateral Throughput on an Idealized Highway", Transportation Science, vol. 29, 1995, pp. 118-127.

[6] J. V. Medanic, D. Ramaswamy, W. R. Perkins, R. and Benekohal, "Partitioned Lane Assignment Strategies for Balancing Excess Lane Capacity on AHS", in Proc. 1995 Amer. Contr. Conf., Seattle, Washington, 1995, pp. 3581-3585.

[7] R. W. Hall, and C. Caliskan, "Design and Evaluation of an Automated Highway System with Optimized Lane Assignment", Trans. Rev. - part C, vol. 7, 1999, pp. 1-15.

[8] K. Kim, D. .I. Cho, and J. .V. Medanic, "Lane Assignment Using a Genetic Algorithm in the Automated Highway Systems", in Proc. of the 8th International IEEE Conference on Intelligent Transportation Systems, Austria, 2005, pp. 332-337.

[9] J. Farrel, and M. Barth, The Global Positioning System and Inertial Navigation: Theory and Practice, McGraw-Hill, 1999.

[10] T.-S. Dao, K. Y. K. Leung, C. M. Clark, and J. P. Huissoon, "Cooperative Lane-Level Positioning Using Markov Localization", in Proc. of IEEE ITSC 2006, Toronto, 2006, pp. 1006-1011.

[11] W. H. Press, B. P. Flannery, S. A. Teukolsky, and W. T. Vetterling, Numerical Recipes in C: The Art of Scientific Computing, Cambrige University Press, 1992.

[12] M. V. Aerde, and H. Rakha, "Multivariate Calibration of Single Regime Speed-Flow-Density Relationships", in Proc. of the Vehicle Navigation and Information Systems (VNIS) conference, Seattle, Washington, August, 1995.

[13] H. Rakha, and B. Crowther, "A Comparison of the Greenshields, Pipes, and Van Aerde Car-Following and Traffic Stream Models", Transportation Research Record No. 1802, Traffic Flow Theory and Highway Capacity 2002, pp. 248-262.

[14] VISSIM [Online]. Available: http://www.english.ptv.de/cgibin/traffic/traf_vissim.pl

[15] K. Y. K. Leung, T.-S. Dao, C. M. Clark, and J. P. Huissoon, "Development of a Hardware-in-the-loop Simulator for Inter-Vehicle Communication Application Research", in Proc. of IEEE ITSC 2006, Toronto, 2006, pp. 1286-1291. 